\title{
Uncertainties of the solar wind in-situ velocity measurements
}

\author{
Gogoberidze G.*and Gorgaslidze E. ${ }^{\dagger}$ \\ Ilia State University, 3/5 Cholokashvili ave., 0162 Tbilisi, Georgia
}

\begin{abstract}
We study spectral features of Alfvénic turbulence in fast solar wind. We propose a general, instrument independent method to estimate the uncertainty in velocity fluctuations obtained by in-situ satellite observations in the solar wind. We show that when the measurement uncertainties of the velocity fluctuations are taken into account the less energetic Elsasser spectrum obeys a unique power law scaling throughout the inertial range as prevailing theories of magnetohydrodynamic turbulence predict.
\end{abstract}

Keywords: Solar Wind: Turbulence

\section{Introduction}

In-situ satellite observations of the solar wind magnetic field and bulk flow span several decades in temporal scales and offer a 'natural laboratory' for the study of MHD turbulence (Bruno \& Carbone, 2013). They have been extensively used to test theoretical predictions of MHD turbulence. The Elsasser fields, $\mathbf{Z}^{ \pm}=\mathbf{v} \pm \mathbf{B} / \sqrt{4 \pi \rho}$, where $\mathbf{v}$ and $\mathbf{B}$ are the velocity and magnetic fields, respectively, and $\rho$ is the average density, represent eigen-functions of counter propagating (with respect to the mean magnetic field) Alfvén waves and therefore they are primary fields for the study of incompressible magnetohydrodynamic (MHD) turbulence. Fluctuations in the fast solar wind are strongly imbalanced - there is more power in Alfvén waves propagating outward from the sun than toward it so that the power in $\mathbf{Z}^{+}$typically dominates over that in $\mathbf{Z}^{-}$.

Using high cadence WIND observations Wicks et al. (2010) studied spectral features of the Elsasser variables in the high frequency part of the inertial interval for the first time. They showed that at 1 $\mathrm{AU}$ (in accordance with earlier studies) in the low frequency part of the inertial interval $\left(10^{-3} \mathrm{~Hz}<\right.$ $\left.f<10^{-2} \mathrm{~Hz}\right) \mathbf{Z}^{-}$nearly follows Kolmogorov scaling which at higher frequencies $\left(f>10^{-2} \mathrm{~Hz}\right)$ is more shallow with $\gamma_{-} \approx-1.3$. The absence of single scaling of the subdominant Elsasser field in the entire inertial interval contradicts all recently developed models of strong, anisotropic imbalanced MHD turbulence which predict a single scaling for sub-dominant $\mathbf{Z}^{-}$spectrum. They also are inconsistent with the results of recent high resolution direct numerical simulations of imbalanced MHD turbulence which showed single scaling of the subdominant Elsasser field in the inertial interval (Turner et al., 2012).

Control of observational uncertainty in the in-situ observations is non-trivial, although these errors often have known bounds. There are different challenges for magnetic field and velocity measurements; solar wind velocity observations are intrinsically more uncertain compared to the magnetic field data (Gogoberidze et al., 2012a,b, 2013, 2018, Hnat et al., 2011, Turner et al., 2012). Here we propose an instrument independent method to estimate the uncertainty on velocity field fluctuations directly from the data. We obtain the systematic shift that this uncertainty introduces into observed spectral exponents. We will see that the shallower $\mathbf{Z}^{-}$spectrum at high frequencies can be entirely accounted for by this uncertainty in the velocity data and the observations of the $\mathbf{Z}^{ \pm}$spectra may in fact within

\footnotetext{
*grigol_gogoberidze@iliauni.edu.ge, Corresponding author

†egorgaslidze@gmail.com
} 
ComBAO Author Template

achievable accuracy of the observations, be in agreement with the predictions of theory and numerical simulations.

\section{Data analysis}

We use data obtained by the WIND spacecraft at 3 second resolution. Magnetic field data is provided by the MFI instrument and density and velocity data by the 3DP instrument. We use observations made during a quiet fast stream. The start time of the interval is 06:00 April 06, 2008 and stop time is 12:00 of April 08, 2008 (Gogoberidze et al., 2012b). During this interval the solar wind speed remained above $550 \mathrm{~km} / \mathrm{s}$. The energy of compressive fluctuations was an order of magnitude lower than that of incompressible fluctuations and, consequently, magnetic and velocity fluctuations, being mainly Alfvénic, were dominated by the components perpendicular to the local mean field. The mean field, $\overline{\mathbf{B}}(t, \tau)$, at some time $t$ and on scale $\tau$ is defined as the magnetic field averaged over the interval $[t-\tau, t+2 \tau]$. The fluctuations of the velocity and magnetic field are defined by standard expressions $\delta \mathbf{v}(t, \tau)=\mathbf{v}(t+\tau)-\mathbf{v}(t)$ and $\delta \mathbf{B}(t, \tau)=\mathbf{B}(t+\tau)-\mathbf{B}(t)$, respectively.

There are several sources of uncertainty in the solar wind velocity measurements (Gogoberidze et al., 2013, Podesta et al., 2002, Turner et al., 2012). The first source is the uncertainty in assessment of the proton distribution function. In addition, in common with all velocity in situ observations, the $3 \mathrm{~s}$ velocity observations on WIND are quantized before ground transmission and this quantization results in high frequency noise or quantization noise. These contributions to observational uncertainty decorrelate the velocity and magnetic field fluctuations at high frequencies. White, delta correlated noise provides a reasonable, instrument independent model for the uncertainty (Podesta et al., 2002). Any measurement of a velocity component fluctuation $\delta v_{o}$ can then be represented as a sum of the 'real' turbulent signal $\delta v_{s}$ and a noise $\delta v_{n}$ which has zero mean and standard deviation $\varepsilon$, so $\delta v_{o}=$ $\delta v_{s}+\delta v_{n}$. Note that the r.m.s. value of a single velocity measuremant $v_{n}$ used to characterise velocity uncertainties in other studies (Podesta et al., 2002, Wicks et al., 2010) is, in our notations, $\varepsilon / \sqrt{2}$. In what follows we will neglect the uncertainties in the magnetic field measurements since generally these are small relative to those of the velocity measurements.

We will first quantify the velocity uncertainty from the data. We will exploit the fact that both the turbulent signal and the noise are random variables with distinct characteristic autocorrelation time scales. We make a key assumption- that the autocorrelation timescale of the underlying turbulent signal is that observed in the magnetic field component fluctuations $\delta B_{o}$ (they have negligible noise) and that this is also the autocorrelation timescale of the 'true' turbulent velocity component fluctuations $\delta v_{s}$. Any difference in the autocorrelation functions of the observed $\delta v_{o}$ and $\delta B_{o}$ are thus attributable to the (delta correlated) noise $\delta v_{n}$ on the velocity. The autocorrelation coefficient (AC) of

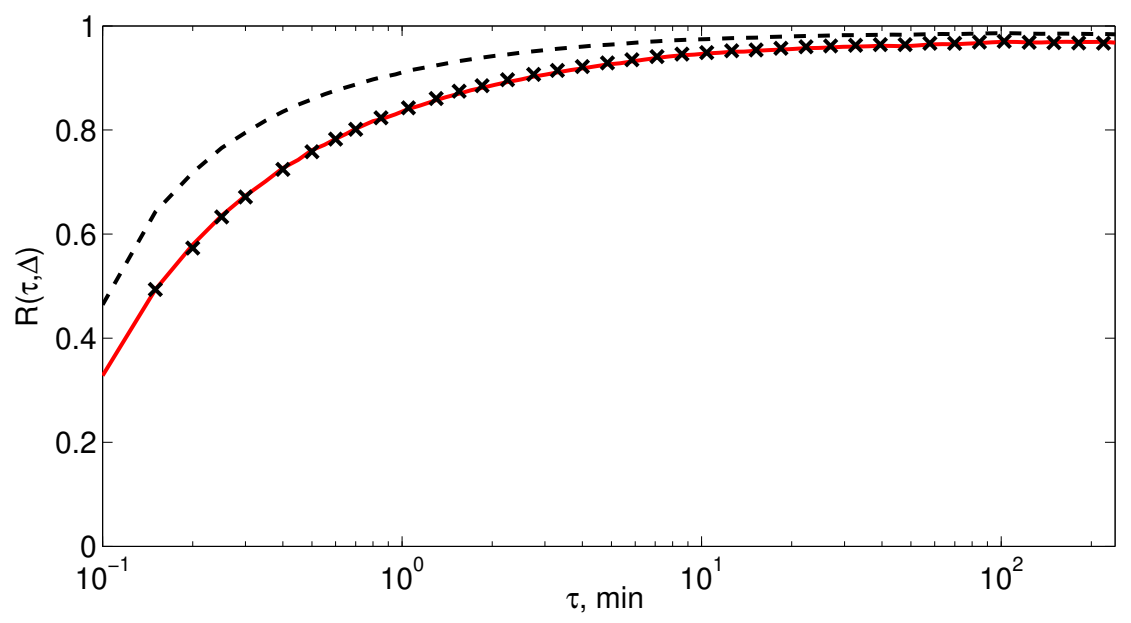

Figure 1. Autocorrelation functions $R_{\delta B_{y}}(\tau, \Delta)$ (black dashed line) of the GSE $y$ component of the magnetic field fluctuation and $R_{\delta v_{y}}(\tau, \Delta)$ (red solid line) with the time lag $\Delta=3 \mathrm{~s}$. 
a component $\delta v$ on time lag $\Delta$ is $R_{\delta v}(\tau, \Delta) \equiv\langle\delta v(t+\Delta, \tau) \delta v(t, \tau)\rangle /\left\langle\delta v(t, \tau)^{2}\right\rangle$, where angular brackets denote time averages with respect to entire studied interval (i.e., with respect to all possible values of $t$ ). The autocorrelation coefficients $R_{\delta B_{o}}(\tau, \Delta)$ and $R_{\delta v_{o}}(\tau, \Delta)$ for GSE $y$ components of the magnetic field and velocity fluctuations are plotted in Figure 1 for lag $\Delta=3 \mathrm{~s}$ as a function of scale $\tau$ with black solid and red dashed lines respectively. We see that the AC grows with scale $\tau$ for both signals and that the velocity AC is systematically lower that that of the magnetic field, consistent with the assumption of uncorrelated noise that principally affects the velocity signal. Given these assumptions one can construct a modelled noisy signal by adding uncorrelated noise to the magnetic field observations. The modelled noisy fluctuations $\delta B_{o+n}=\delta B_{o}+\delta B_{n}$, where $\delta B_{n}$ are delta correlated Gaussian distributed random numbers with zero mean and standard deviation $\varepsilon_{B}$. The magnitude of the modelled noise $\varepsilon_{B}$ can then be systematically varied. We first verify that this simple noise model is sufficient to reproduce the $\mathrm{AC}$ as a function of $\tau$ for the velocity fluctuations. The AC of the modelled $\delta B_{o+n}$ is shown by the black crosses in Fig. 1 and this can be seen to closely coincide with the observed $\mathrm{AC}$ of velocity fluctuations $\delta v_{o}$. Assuming Alfvénic fluctuations the magnitude of $\varepsilon_{B}$ used to generate this curve corresponds to an uncertainty in the velocity fluctuations of

$$
\varepsilon_{v} \equiv \varepsilon_{B} \sqrt{\frac{\left\langle\delta v_{o}^{2}\right\rangle}{\left\langle\delta B_{o+n}^{2}\right\rangle}}=4 \mathrm{~km} / \mathrm{s} .
$$

Hereafter in this paper $\varepsilon$ denotes r.m.s. value of observed velocity fluctuation $\delta v$, whereas $\varepsilon_{v}$ denotes its estimate, derived using different methods described in the paper. Thus, $\varepsilon \sim 4 \mathrm{~km} / \mathrm{s}$ (which corresponds to the uncertainty of a single velocity component of $\varepsilon / \sqrt{2} \sim 2.83 \mathrm{~km} / \mathrm{s}$ ) is a reasonable estimate of the amplitude of the noise on the turbulent velocity signal. We will develop this idea to obtain a general method to estimate the uncertainty direct from the data. In the next section we will compare our assessment of the velocity measurement uncertainties with the estimate derived in other studies. First, we will see how these uncertainties can affect measurements of scaling exponents and the conclusions that can be drawn from them. In Figure 2 we plot the observed second order

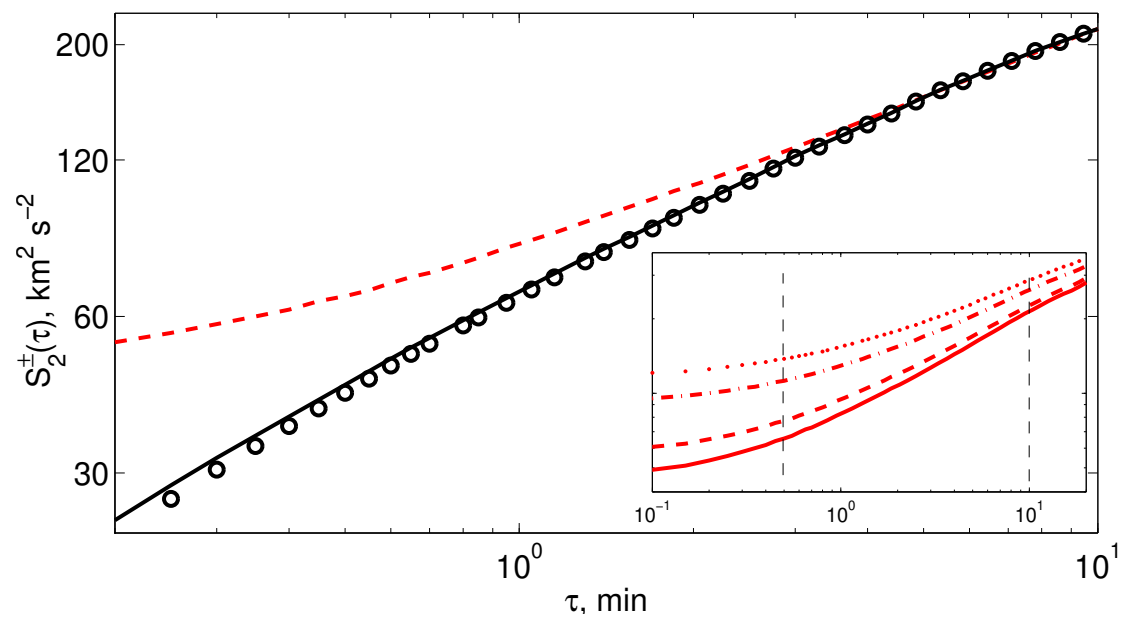

Figure 2. The normalized second order structure functions: of the GSE $z$ component of the subdominant Elsasser variable $Z_{z}^{-}$(red dashed line), of the dominant Elsasser variable $Z_{z}^{+}$(black solid line) and $S_{2}^{-}$(circles, see text for details). In the insert: the second order structure functions of the sub-dominant Elsasser variable for different values of added Gaussian noise. Raw observations are denoted by the solid line, and added noise is equivalent to $\varepsilon_{v}=2 \mathrm{~km} / \mathrm{s}$ (dashed line), $\varepsilon_{v}=4 \mathrm{~km} / \mathrm{s}$ (dash-dotted line) and $\varepsilon_{v}=5 \mathrm{~km} / \mathrm{s}$ (dotted line).

structure functions $S_{2}$ of a component of the fluctuations in the Elsasser variables, where the Elsasser components are given by $\delta Z_{i}^{ \pm}(\tau)=\delta v_{i}(t, \tau) \pm \delta B_{i}(t, \tau) / \sqrt{4 \pi \bar{\rho}}$ and $\bar{\rho}(\tau)$ is the local mean value of the density averaged over the time scale of the fluctuations $\tau$, i.e., over the interval $[t, t+\tau]$, and $S_{2}^{ \pm}=\left\langle\delta Z_{i}^{ \pm}(\tau)^{2}\right\rangle$. The solid lines are the structure functions of GSE $z$ components of the dominant $\delta Z_{z}^{+}$(black solid line) and subdominant $\delta Z_{z}^{-}$(red dashed line) fields. They are normalized to have the 
same values at $\tau=10 \mathrm{~min}$ scale on this plot; the power in $\delta Z_{z}^{+}$is 20 times that in $\delta Z_{z}^{-}$at $\tau=10 \mathrm{~min}$ scale. For the ideal statistical scaling of fully developed MHD turbulence we anticipate the scaling $S_{2} \sim \tau^{\zeta_{ \pm}(2)}$ and turbulence theories predict constant values of $\zeta^{ \pm}(2)$ over the entire inertial interval (they are directly related to the power spectral exponents $\gamma_{ \pm}$via $\gamma_{ \pm}=-\zeta_{ \pm}(2)-1$ ). We can see that, consistent with earlier studies (e.g., Wicks et al. (2010)), the subdominant Elsasser variable does not follow a single power law in the inertial interval. A linear mean least square fit on log-log plot over scales $30 \mathrm{~s}<\tau<10$ min gives $\gamma_{+}=-1.54 \pm 0.02$ and $\gamma_{-}=-1.40 \pm 0.02$, consistent with previous observations (Bruno \& Carbone, 2013, Wicks et al., 2010).

A quantitative demonstration of the effect of noise is provided by calculating $S_{2}^{-}\left(\delta B_{o+n}, \delta v_{o}\right) \equiv$ $\left\langle\left[\delta v_{i, o}-\left(\delta B_{i, o}+\delta B_{i, n}\right) / \sqrt{4 \pi \bar{\rho}}\right]^{2}\right\rangle$ which for uncorrelated noise is equivalent to $S_{2}^{-}\left(\delta B_{o}, \delta v_{o}\right)+\varepsilon_{v}^{2}$. Here a different 'Alfvénic' relation, $\varepsilon_{v}=\varepsilon_{B} / \sqrt{4 \pi \bar{\rho}}$, is used to relate the velocity and magnetic field uncertainties. This is shown in the inset of Figure 2 for a range of amplitudes of $\delta B_{n}$, which are equivalent to velocity noise uncertainties of $\varepsilon_{v}=2,4$ and $5 \mathrm{~km} / \mathrm{s}$. We can see that addition of 'white' (delta correlated) noise always systematically 'flattens' these curves, that is, it decreases the value of the scaling exponent; for $\varepsilon_{v}=4 \mathrm{~km} / \mathrm{s}$ pseudo noise strongly affects $S_{2}^{-}$at all scales in the inertial interval. The 'flattening' of the pseudo-noisy $S_{2}^{-}\left(\delta B_{o+n}, \delta v_{o}\right)$ curve, that is, the change in the mean exponent over timescales $30 \mathrm{~s}<\tau<10 \mathrm{~min}$ is $\Delta \gamma_{-} \approx 0.13$, is close to the observed difference between exponents of the dominant and subdominant fields $\left(\gamma_{-}-\gamma_{+}=0.14\right)$ hence this difference could be just due to noise in the velocity data.

We now obtain an estimate of the structure function in the absence of the noise in the velocity, $S_{2}^{-}\left(\delta B_{o}, \delta v_{s}\right) \equiv\left\langle\left(\delta Z_{s}^{-}\right)^{2}\right\rangle$, where $\delta Z_{s}^{-}=\delta v_{s}-\delta B_{o} / \sqrt{4 \pi \bar{\rho}}$. We have that the observed structure function $S_{2}^{-}\left(\delta B_{o}, \delta v_{o}\right)=\left\langle\left(\delta Z_{s}^{-}+\delta v_{n}\right)^{2}\right\rangle=S_{2}^{-}\left(\delta B_{o}, \delta v_{s}\right)+2\left\langle\delta Z_{s}^{-} \delta v_{n}\right\rangle+\varepsilon^{2}$. Assuming as before that the turbulent signal and the velocity error are uncorrelated $\left(\left\langle\delta Z_{s}^{-} \delta v_{n}\right\rangle=0\right)$ we obtain $S_{2}^{-}\left(\delta B_{o}, \delta v_{s}\right)=$ $S_{2}^{-}\left(\delta B_{o}, \delta v_{o}\right)-\varepsilon^{2}$. The plot of our estimated $S_{2}^{-}\left(\delta B_{o}, \delta v_{s}\right)$ is given by black circles in Figure 2 for $\varepsilon=4 \mathrm{~km} / \mathrm{s}$. This error compensated subdominant $S_{2}^{-}\left(\delta B_{o}, \delta v_{s}\right)$ curve now has a single scaling throughout the inertial range, consistent with current theories and numerical predictions (Podesta et al., 2002). We can also see that the slope of $S_{2}^{-}\left(\delta B_{o}, \delta v_{s}\right)$ coincides quite closely with the observed slope of the dominant Elsasser variable $S_{2}^{+}$. The uncertainty in the velocity that we have estimated from the data is thus sufficient to account for the departure in scaling between the $\delta \mathbf{Z}^{-}$and $\delta \mathbf{Z}^{+}$ Elsasser variables and these observations may in fact within the achievable accuracy be in agreement with theories (Podesta et al., 2002) that predict a single scaling for $\delta \mathbf{Z}^{-}$and $\delta \mathbf{Z}^{+}$.

\section{Conclusions}

In summary, we have presented a general, instrument independent method to determine uncertainty in the velocity fluctuations in single point measurements. We have shown that this uncertainty is sufficient to account for both the absence of single scaling of the subdominant Elsasser field and for the difference of $\mathbf{Z}^{ \pm}$slopes in the inertial interval. Thus, our findings are able to report for the first time that the observations are, within the achievable accuracy, in agreement with the predictions of theory and numerical simulations.

\section{Acknowledgements}

This work has been supported by Shota Rustaveli National Science Foundation grant FR-18-19964.

\section{References}

Bruno R., Carbone V., 2013, Liv. Rev. Sol. Phys., 10, 2

Gogoberidze G., Chapman S. C., Hnat B., 2012a, Phys. Plasmas, 19, 102310

Gogoberidze G., Chapman S. C., Hnat B., Dunlop M. W., 2012b, MNRAS, 426, 951

Gogoberidze G., Perri S., Carbone V., 2013, ApJ, 769, 111

Gogoberidze G., Voitenko Y., Machabeli G., 2018, MNRAS, 480, 1864 
Hnat B., Chapman S. C., Gogoberidze G., Wicks R. T., 2011, PRE, 84, 065401

Podesta J. J., Chandran B. D. J., Bhattacharjee A., Roberts D. A., Goldstein M. L., 2002, GRL, 29, 1839

Turner A. J., Gogoberidze G., Chapman S. C., 2012, PRL, 108, 085001

Wicks R. T., Horbury T. S., Chen C. H. S., ZastenkerSchekochihin A. A., 2010, MNRAS, 407, L31 